\title{
Mass Spectrum of a Baryon Octet is Linear
}

\author{
L. Burakovsky* \\ Theoretical Division, T-8 \\ Los Alamos National Laboratory \\ Los Alamos NM 87545, USA \\ and \\ L.P. Horwitz ${ }^{\dagger}$ \\ School of Natural Sciences \\ Institute for Advanced Study \\ Princeton NJ 08540, USA
}

\begin{abstract}
It is argued that the mass spectrum of a baryon octet is linear, consistent with a Gell-Mann-Okubo type relation for the squared masses of the baryons obtained previously by Balázs and Nicolescu. The mass spectrum of a baryon decuplet is briefly discussed.
\end{abstract}

Key words: hadronic resonance spectrum, Gell-Mann-Okubo

PACS: 12.40.Ee, 12.40.Yx, 14.20.-c

\footnotetext{
*Bitnet: BURAKOV@QCD.LANL.GOV

$\dagger$ Bitnet: HORWITZ@SNS.IAS.EDU. On sabbatical leave from School of Physics and Astronomy, Tel Aviv University, Ramat Aviv, Israel. Also at Department of Physics, Bar-Ilan University, RamatGan, Israel
} 
The hadronic mass spectrum is an essential ingredient in theoretical investigations of the physics of strong interactions. It is well known that the correct thermodynamic description of hot hadronic matter requires consideration of higher mass excited states, the resonances, whose contribution becomes essential at temperatures $\sim O(100 \mathrm{MeV})$ [1, 2]. The method for taking into account these resonances was suggested by Belenky and Landau [3] as considering unstable particles on an equal footing with the stable ones in the thermodynamic quantities; e.g., the formulas for the pressure and energy density in a resonance gas read']

$$
\begin{gathered}
p=\sum_{i} p_{i}=\sum_{i} g_{i} \frac{m_{i}^{2} T^{2}}{2 \pi^{2}} K_{2}\left(\frac{m_{i}}{T}\right), \\
\rho=\sum_{i} \rho_{i}, \quad \rho_{i}=T \frac{d p_{i}}{d T}-p_{i},
\end{gathered}
$$

where $g_{i}$ are the corresponding degeneracies ( $J$ and $I$ are spin and isospin, respectively),

$$
g_{i}=\frac{\pi^{4}}{90} \times\left[\begin{array}{ll}
\left(2 J_{i}+1\right)\left(2 I_{i}+1\right) & \text { for non }- \text { strange mesons } \\
4\left(2 J_{i}+1\right) & \text { for strange }(K) \text { mesons } \\
2\left(2 J_{i}+1\right)\left(2 I_{i}+1\right) \times 7 / 8 & \text { for baryons }
\end{array}\right.
$$

These expressions may be rewritten with the help of a resonance spectrum,

$$
\begin{gathered}
p=\int_{m_{1}}^{m_{2}} d m \tau(m) p(m), \quad p(m) \equiv \frac{m^{2} T^{2}}{2 \pi^{2}} K_{2}\left(\frac{m}{T}\right), \\
\rho=\int_{m_{1}}^{m_{2}} d m \tau(m) \rho(m), \quad \rho(m) \equiv T \frac{d p(m)}{d T}-p(m),
\end{gathered}
$$

normalized as

$$
\int_{m_{1}}^{m_{2}} d m \tau(m)=\sum_{i} g_{i}
$$

where $m_{1}$ and $m_{2}$ are the masses of the lightest and heaviest species, respectively, entering the formulas (1),(2).

In both the statistical bootstrap model [4, [5] and the dual resonance model [6], a resonance spectrum takes on the form

$$
\tau(m) \sim m^{a} e^{m / T_{0}},
$$

where $a$ and $T_{0}$ are constants. The treatment of a hadronic resonance gas by means of the spectrum (6) leads to a singularity in the thermodynamic functions at $T=T_{0}$

\footnotetext{
${ }^{1}$ For simplicity, we neglect the chemical potential and approximate the particle statistics by the Maxwell-Boltzmann one.
} 
[4, 5] and, in particular, to an infinite number of the effective degrees of freedom in the hadron phase, thus hindering a transition to the quark-gluon phase. Moreover, as shown by Fowler and Weiner [7], an exponential mass spectrum of the form (6) is incompatible with the existence of the quark-gluon phase: in order that a phase transition from the hadron phase to the quark-gluon phase be possible, the hadronic spectrum cannot grow with $m$ faster than a power.

In our previous work [8] we considered a model for a transition from a phase of strongly interacting hadron constituents, described by a manifestly covariant relativistic statistical mechanics which turned out to be a reliable framework in the description of realistic physical systems [9], to the hadron phase described by a resonance spectrum, Eqs. (3),(4). An example of such a transition may be a relativistic high temperature Bose-Einstein condensation studied by the authors in ref. [10], which corresponds, in the way suggested by Haber and Weldon [11], to spontaneous flavor symmetry breakdown, $S U(3)_{F} \rightarrow S U(2)_{I} \times U(1)_{Y}$, upon which hadronic multiplets are formed, with the masses obeying the Gell-Mann-Okubo formulas 12

$$
m^{\ell}=a+b Y+c\left[\frac{Y^{2}}{4}-I(I+1)\right] ;
$$

here $I$ and $Y$ are the isospin and hypercharge, respectively, $\ell$ is 2 for mesons and 1 for baryons, and $a, b, c$ are independent of $I$ and $Y$ but, in general, depend on $(p, q)$, where $(p, q)$ is any irreducible representation of $S U(3)$. Then only the assumption on the overall degeneracy being conserved during the transition is required to lead to the unique form of a resonance spectrum in the hadron phase:

$$
\tau(m)=C m, \quad C=\text { const. }
$$

Zhirov and Shuryak [13] have found the same result on phenomenological grounds. As shown in ref. [13], the spectrum (8), used in the formulas (3),(4) (with the upper limit of integration infinity), leads to the equation of state $p, \rho \sim T^{6}, p=\rho / 5$, called by Shuryak the "realistic" equation of state for hot hadronic matter [1], which has some experimental support. Zhirov and Shuryak [13] have calculated the velocity of sound, $c_{s}^{2} \equiv d p / d \rho=c_{s}^{2}(T)$, with $p$ and $\rho$ defined in Eqs. (1),(2), and found that $c_{s}^{2}(T)$ at first increases with $T$ very quickly and then saturates at the value of $c_{s}^{2} \simeq 1 / 3$ if only the pions are taken into account, and at $c_{s}^{2} \simeq 1 / 5$ if resonances up to $M \sim 1.7$ $\mathrm{GeV}$ are included.

In order to understand why a linear spectrum is the actual spectrum of a hadronic multiplet, one may restrict himself to one family of the Regge trajectories (e.g., those on which the members of the baryon $J^{P}=\frac{1}{2}^{+}, \frac{3}{2}^{-}, \frac{5}{2}^{+}, \ldots$ octets lie), and calculate the sum in Eq. (1) in two different ways:

1. Fix spin and calculate the sum in Eq. (1) for an individual multiplet, by the introduction of the multiplet spectrum, $\tau(m)$, as in Eq. (3); then with a spin 
degeneracy, $2 J_{i}+1 \simeq 2 J_{i} \simeq 2 \alpha^{\prime} m_{i}^{2}$, involved, $\alpha^{\prime} \cong 0.84 \mathrm{GeV}^{-2}$ being a universal Regge slope, the sum (1) will reduce to Eq. (3) with [8]

$$
\tau^{\prime}(m) \sim m^{2} \tau(m)
$$

where $\tau^{\prime}(m)$ is the mass spectrum of a family of the Regge trajectories.

2. Fix isospin and calculate the sum (1) for an individual trajectory, then multiply the result by the number of the trajectories for an individual multiplet (e.g., 8 for a baryon octet). Since the squared masses of the states lying on a trajectory, are $m_{0}^{2}, m_{0}^{2}+1 / \alpha^{\prime}, m_{0}^{2}+2 / \alpha^{\prime}, \ldots$, the sum (1) may be calculated directly, using the Euler-Maclaurin summation formula

$$
\sum_{n=n_{1}}^{n_{2}} f_{n}=\int_{n_{1}}^{n_{2}} d n f(n)+\frac{1}{2}\left[f\left(n_{1}\right)+f\left(n_{2}\right)\right]+(\text { derivative terms })
$$

resulting in Eq. (3) with [8]

$$
\tau^{\prime}(m) \sim m^{3}
$$

It then follows from $(9),(11)$ that

$$
\tau(m) \sim m
$$

i.e., the mass spectrum of an individual hadronic multiplet is linear.

We have checked the coincidence of the results given by the linear spectrum (8) with those obtained directly from Eq. (1) for the actual hadronic species with the corresponding degeneracies, for all well-established hadronic multiplets, both mesonic and baryonic, and found it excellent [8]. Shown are typical figures of ref. [8] in which the results given by both, Eq. (1), and Eq. (3) with a linear spectrum, are compared, for the following three well-established baryon octets:

$$
\begin{aligned}
& J^{P}=\frac{1}{2}^{+}, N(939), \Lambda(1116), \Sigma(1190), \Xi(1320) \\
& J^{P}=\frac{3}{2}^{-}, N(1520), \Lambda(1690), \Sigma(1670), \Xi(1823) \\
& J^{P}=\frac{5}{2}^{+}, N(1680), \Lambda(1820), \Sigma(1915), \Xi(2030)
\end{aligned}
$$

Thus, the theoretical implication that a linear spectrum is the actual spectrum in the description of individual hadronic multiplets, is consistent with experiment as well. In our recent papers 114, 15] we have shown that the linear spectrum of an individual meson nonet is consistent with the Gell-Mann-Okubo mass formula (as follows from (7))

$$
m_{1}^{2}+3 m_{8}^{2}=4 m_{1 / 2}^{2}
$$

(in fact, this formula may be derived with the help of a linear spectrum [14), and leads to an extra relation for the masses of the isoscalar states, $m_{0^{\prime}}$ and $m_{0^{\prime \prime}}$, (of

\footnotetext{
${ }^{2}$ Instead of a direct comparison of Eqs. (1) and (3), we compared the expressions $p / p_{S B}$ for both cases, where $p_{S B} \equiv \sum_{i} g_{i} \pi^{2} / 90 T^{4}$, i.e., $p_{S B}$ is the pressure in an ultrarelativistic gas with $g=\sum_{i} g_{i}$ degrees of freedom.
} 
which 0 ' belongs to a mostly octet),

$$
m_{0^{\prime}}^{2}+m_{0^{\prime \prime}}^{2}=m_{0}^{2}+m_{8}^{2}=2 m_{1 / 2}^{2},
$$

with $m_{1}, m_{1 / 2}, m_{8}, m_{0}$ being the masses of the isovector, isospinor, and isoscalar octet and singlet states, respectively, which for an almost ideally mixed nonet reduces to 14, 15

$$
m_{0^{\prime \prime}}^{2} \simeq m_{1}^{2}, \quad m_{0^{\prime}}^{2} \simeq 2 m_{1 / 2}^{2}-m_{1}^{2} .
$$

The relation (14) was checked in ref. 115 and shown to hold with an accuracy of up to $\sim 3 \%$ for all well-established nonets. In ref. [14] we have generalized a linear spectrum to the case of four quark flavors and derived the corresponding Gell-MannOkubo mass formula for an $S U(4)$ meson hexadecuplet, in good agreement with the experimentally established masses of the charmed mesons. In ref. [16] we have applied a linear spectrum to the problem of establishing the correct $q \bar{q}$ assignment for the problematic meson nonets, like the scalar, axial-vector and tensor ones, and separating out non- $q \bar{q}$ mesons.

By applying the arguments of refs. [14, 15], one may show that the linear spectrum of a baryon octet leads to a Gell-Mann-Okubo type relation for the squared masses of the baryons. Indeed, for a baryon octet, one has 8 isospin states: $2 N, 1 \Lambda, 3 \Sigma$ and $2 \Xi$, the $\Lambda$ and $\Sigma$ states being mass degenerate on a naive quark model level, since both are composed of two light $u$ - and $d$-quarks and one heavier $s$-quark: $m_{\Lambda}=m_{\Sigma} \equiv m_{\Sigma^{\prime}}$. The average mass squared of an octet should coincide with that calculated with the help of a linear spectrum 114, 15; hence

$$
\frac{2 m_{N}^{2}+4 m_{\Sigma^{\prime}}^{2}+2 m_{\Xi}^{2}}{8}=\frac{m_{N}^{2}+m_{\Xi}^{2}}{2},
$$

and therefore, $m_{N}^{2}+m_{\Xi}^{2}=2 m_{\Sigma^{\prime}}^{2}$, or

$$
m_{\Xi}^{2}-m_{\Sigma^{\prime}}^{2}=m_{\Sigma^{\prime}}^{2}-m_{N}^{2}
$$

Let us check the relation (17) for the three well-established baryon octets indicated above. We shall take $m_{\Sigma^{\prime}}=\left(m_{\Lambda}+m_{\Sigma}\right) / 2$, with $m_{\Lambda}$ and $m_{\Sigma}$ being the masses of the physical $\Lambda$ and $\Sigma$ states, respectively.

1. $J^{P}=\frac{1}{2}^{+}$octet. In this case $m_{\Sigma^{\prime}}=1150 \mathrm{MeV}$, and one has $0.42 \mathrm{GeV}^{2}$ on the l.h.s. of Eq. (17) vs. $0.44 \mathrm{GeV}^{2}$ on the r.h.s.

2. $J^{P}=\frac{3}{2}^{-}$octet. Here $m_{\Sigma^{\prime}}=1680 \mathrm{MeV}$, and one has $0.50 \mathrm{GeV}^{2}$ on the 1.h.s. of Eq. (17) vs. $0.51 \mathrm{GeV}^{2}$ on the r.h.s.

3. $J^{P}=\frac{1}{2}^{+}$octet. In this case $m_{\Sigma^{\prime}}=1865 \mathrm{MeV}$, and one has $0.64 \mathrm{GeV}^{2}$ on the l.h.s. of (17) vs. $0.655 \mathrm{GeV}^{2}$ on the r.h.s.

One sees that for the well-established baryon octets, the relation (17) holds with a high accuracy, as for the standard (linear in mass) Gell-Mann-Okubo formula (as follows from (7))

$$
\frac{m_{N}+m_{\Xi}}{2}=\frac{3 m_{\Lambda}+m_{\Sigma}}{4}
$$


which for these octets may be shown to hold with an accuracy of up to $\sim 1 \%$.

The formula (17), together with the relation (as follows from (15))

$$
m_{0^{\prime}}^{2}-m_{1 / 2}^{2}=m_{1 / 2}^{2}-m_{1^{\prime}}^{2}, \quad m_{1^{\prime}} \equiv\left(m_{1}+m_{0^{\prime \prime}}\right) / 2,
$$

suggest the empirical rule for the splitting of the squared masses of the hadrons containing zero, one, and two $s$ - and/or $\bar{s}$-quarks, first arrived at by Balázs and Nicolescu using two different approaches to the confinement region of hadronic physics described in refs. [17, 18]: [3]

$$
m_{2 s}^{2}-m_{1 s}^{2}=m_{1 s}^{2}-m_{0 s}^{2} .
$$

If one assumes that this relation may be generalized to include also the hadron containing three $s$ - or $\bar{s}$-quarks, as follows:

$$
m_{3 s}^{2}-m_{2 s}^{2}=m_{2 s}^{2}-m_{1 s}^{2}=m_{1 s}^{2}-m_{0 s}^{2},
$$

one may apply it to a baryon decuplet. The only decuplet well-established experimentally is $J^{P}=\frac{3}{2}^{+}, \Delta(1232), \Sigma(1385), \Xi(1534), \Omega(1672)$. For this decuplet, Eq. (20) gives

$$
\begin{aligned}
& m_{3 s}^{2}-m_{2 s}^{2}=m_{\Omega}^{2}-m_{\Xi}^{2}=0.44 \mathrm{GeV}^{2} \\
& m_{2 s}^{2}-m_{1 s}^{2}=m_{\Xi}^{2}-m_{\Sigma}^{2}=0.435 \mathrm{GeV}^{2} \\
& m_{2 s}^{2}-m_{1 s}^{2}=m_{\Sigma}^{2}-m_{\Delta}^{2}=0.40 \mathrm{GeV}^{2}
\end{aligned}
$$

One sees that the relation (20) holds for the baryon $J^{P}=\frac{3}{2}^{+}$decuplet with an accuracy which is not lower than that of the standard Gell-Mann-Okubo mass formula (as follows from (7))

$$
m_{\Omega}-m_{\Xi}=m_{\Xi}-m_{\Sigma}=m_{\Sigma}-m_{\Delta}
$$

We note that with the values $m_{N}=0.95 \mathrm{GeV}, m_{\Delta}=1.22 \mathrm{GeV}$, indicated in [17], in good agreement with experiment, the relations (17) and (20) become almost exact for the baryon $J^{P}=\frac{1}{2}^{+}$octet and $J^{P}=\frac{3}{2}^{+}$decuplet, respectively: now one has 0.42 $\mathrm{GeV}^{2}$ on both sides of Eq. (17), and $m_{\Sigma}^{2}-m_{\Delta}^{2}=0.43 \mathrm{GeV}^{2}$ in Eq. (20).

Finally, let us briefly discuss the mass spectrum of a baryon decuplet. According to the arguments given above, the mass spectrum of a baryon decuplet is linear,

\footnotetext{
${ }^{3}$ The relation (19) holds for a close-to-ideally mixed meson nonet, where $0^{\prime} \simeq s \bar{s}$ is composed almost of the $s$-quark and its antiquark, and $0^{\prime \prime} \simeq(u \bar{u}+d \bar{d}) / \sqrt{2}$ is composed almost of two light quark and antiquark and is mass degenerate with $1=(u \bar{u},(u \bar{u}-d \bar{d}) / \sqrt{2}, d \bar{d})$.
} 
as well as for a meson nonet and a baryon octet. The average mass squared of a decuplet calculated directly for 10 isospin states should, therefore, coincide with the value given by a linear spectrum, i.e.,

$$
\frac{4 m_{\Delta}^{2}+3 m_{\Sigma}^{2}+2 m_{\Xi}^{2}+m_{\Omega}^{2}}{10}=\frac{m_{\Delta}^{2}+m_{\Omega}^{2}}{2},
$$

leading to the relation

$$
3 m_{\Sigma}^{2}+2 m_{\Xi}^{2}=m_{\Delta}^{2}+4 m_{\Omega}^{2},
$$

which, however, does not hold. The reason is that 10 isospin degrees of freedom of the decuplet are distributed with a linear spectrum in a mass interval which is broader than $\left(m_{\Delta}, m_{\Omega}\right)$. Indeed, assuming the validity of Eq. (20), i.e., $m_{\Sigma}^{2}=m_{\Delta}^{2}+a$, $m_{\Xi}^{2}=m_{\Delta}^{2}+2 a, m_{\Omega}^{2}=m_{\Delta}^{2}+3 a, a \simeq 0.43 \mathrm{GeV}^{2}$, it then follows that

$$
\left\langle m^{2}\right\rangle=\frac{4 m_{\Delta}^{2}+3 m_{\Sigma}^{2}+2 m_{\Xi}^{2}+m_{\Omega}^{2}}{10}=m_{\Delta}^{2}+a=m_{\Sigma}^{2} ;
$$

one therefore finds that the mass interval of the decuplet is $\left(\sqrt{m_{\Delta}^{2}-a}, m_{\Omega}\right)$, and the average mass squared given by a linear spectrum for this interval coincides with (24):

$$
\frac{1}{2}\left(m_{\Delta}^{2}-a+m_{\Delta}^{2}+3 a\right)=m_{\Delta}^{2}+a=m_{\Sigma}^{2} .
$$

Fig. 4 shows the ratio $p / p_{S B}$ calculated from both, Eq. (1) with the actual baryon masses and degeneracies, and a linear spectrum with the mass interval of the decuplet $\left(1.04 \mathrm{GeV} \cong \sqrt{m_{\Delta}^{2}-a}, 1.672 \mathrm{GeV}=m_{\Omega}\right)$. One sees that the results coincide.

The generalization of a linear spectrum of a baryon multiplet to the case of four quark flavors and the derivation of the corresponding Gell-Mann-Okubo mass formula for an $S U(4)$ baryon 20-plet will be given in a separate publication.

\section{Acknowledgements}

One of us (L.B.) wish to thank E.V. Shuryak for very valuable discussions on hadronic resonance spectrum.

\section{References}

[1] E.V. Shuryak, The QCD Vacuum, Hadrons and the Superdense Matter, (World Scientific, Singapore, 1988)

[2] C.R. Alcock, G.M. Fuller and G.J. Mathews, Astrophys. J. 320 (1987) 439

H. Bebie, P. Gerber, J.L. Goity and H. Leutwyler, Nucl. Phys. B 378 (1992) 95 
[3] S.Z. Belenky and L.D. Landau, Sov. Phys. Uspekhi 56 (1955) 309; Nuovo Cim. Suppl. 3 (1956) 15

[4] R. Hagedorn, Nuovo Cim. 35 (1965) 216; Nuovo Cim. Suppl. 6 (1968) 169, 311

[5] S. Frautschi, Phys. Rev. D 3 (1971) 2821

[6] S. Fubini and G. Veneziano, Nuovo Cim. A 64 (1969) 811

[7] G.N. Fowler and R.M. Weiner, Phys. Lett. B 89 (1980) 394

[8] L. Burakovsky, L.P. Horwitz and W.C. Schieve, Hadronic Resonance Spectrum: Power vs. Exponential Law. Experimental Evidence; to be published

[9] L.P. Horwitz and C. Piron, Helv. Phys. Acta 46 (1973) 316

L.P. Horwitz, W.C. Schieve and C. Piron, Ann. Phys. (N.Y.) 137 (1981) 306

L.P. Horwitz, S. Shashoua and W.C. Schieve, Physica A 161 (1989) 300

L. Burakovsky, Manifestly Covariant Relativistic Statistical Mechanics as a Framework for Description of Realistic Physical Systems, Ph.D. thesis (Tel-Aviv University, 1995), unpublished; L. Burakovsky, L.P. Horwitz and W.C. Schieve, Mass - Proper Time Uncertainty Relation in a Manifestly Covariant Relativistic Statistical Mechanics, to be published

[10] L. Burakovsky, L.P. Horwitz and W.C. Schieve, A New Relativistic High Temperature Bose-Einstein Condensation; Phys. Rev. D, in press

[11] H.A. Haber and H.E. Weldon, Phys. Rev. D 25 (1982) 502 (1994) 4725

[12] S. Okubo, Prog. Theor. Phys. 27 (1962) 949, 28 (1962) 24

M. Gell-Mann and Y. Ne'eman, The Eightfold Way, (Benjamin, N.Y., 1964)

[13] O.V. Zhirov and E.V. Shuryak, Sov. J. Nucl. Phys. 21 (1975) 443

[14] L. Burakovsky and L.P. Horwitz, Gell-Mann-Okubo Mass Formula for SU(4) Meson Hexadecuplet, to be published

[15] L. Burakovsky and L.P. Horwitz, Mass Spectrum of a Meson Nonet is Linear, to be published

[16] L. Burakovsky and L.P. Horwitz, Hadronic Resonance Spectrum May Help in Resolution of Meson Nonet Enigmas; to be published

[17] L.A.P. Balázs and B. Nicolescu, Phys. Rev. D 28 (1983) 2818

[18] L.A.P. Balázs, Phys. Lett. B 120 (1983) 426 


\section{FIGURE CAPTIONS}

Fig. 1. Temperature dependence of the ratio $p / p_{S B}$ as calculated from: a) Eq. (1), b) Eq. (3) with a linear spectrum, for the $J^{P}=\frac{1}{2}^{+}$baryon octet, $N(939), \Lambda(1116)$, $\Sigma(1190), \Xi(1320)$.

Fig. 2. The same as Fig. 1 for the $J^{P}=\frac{3}{2}^{-}$baryon octet, $N(1520), \Lambda(1690)$, $\Sigma(1670), \Xi(1823)$.

Fig. 3. The same as Fig. 1 for the $J^{P}=\frac{5}{2}^{+}$baryon octet, $N(1680), \Lambda(1820)$, $\Sigma(1915), \Xi(2030)$.

Fig. 2. The same as Fig. 1 for the $J^{P}=\frac{3}{2}^{+}$baryon decuplet, $\Lambda(1232), \Sigma(1385)$, $\Xi(1534), \Omega(1672)$, with the mass interval for a linear spectrum $(1.04 \mathrm{GeV}, 1.672 \mathrm{GeV})$. 


$$
1
$$




$$
\text { L }
$$




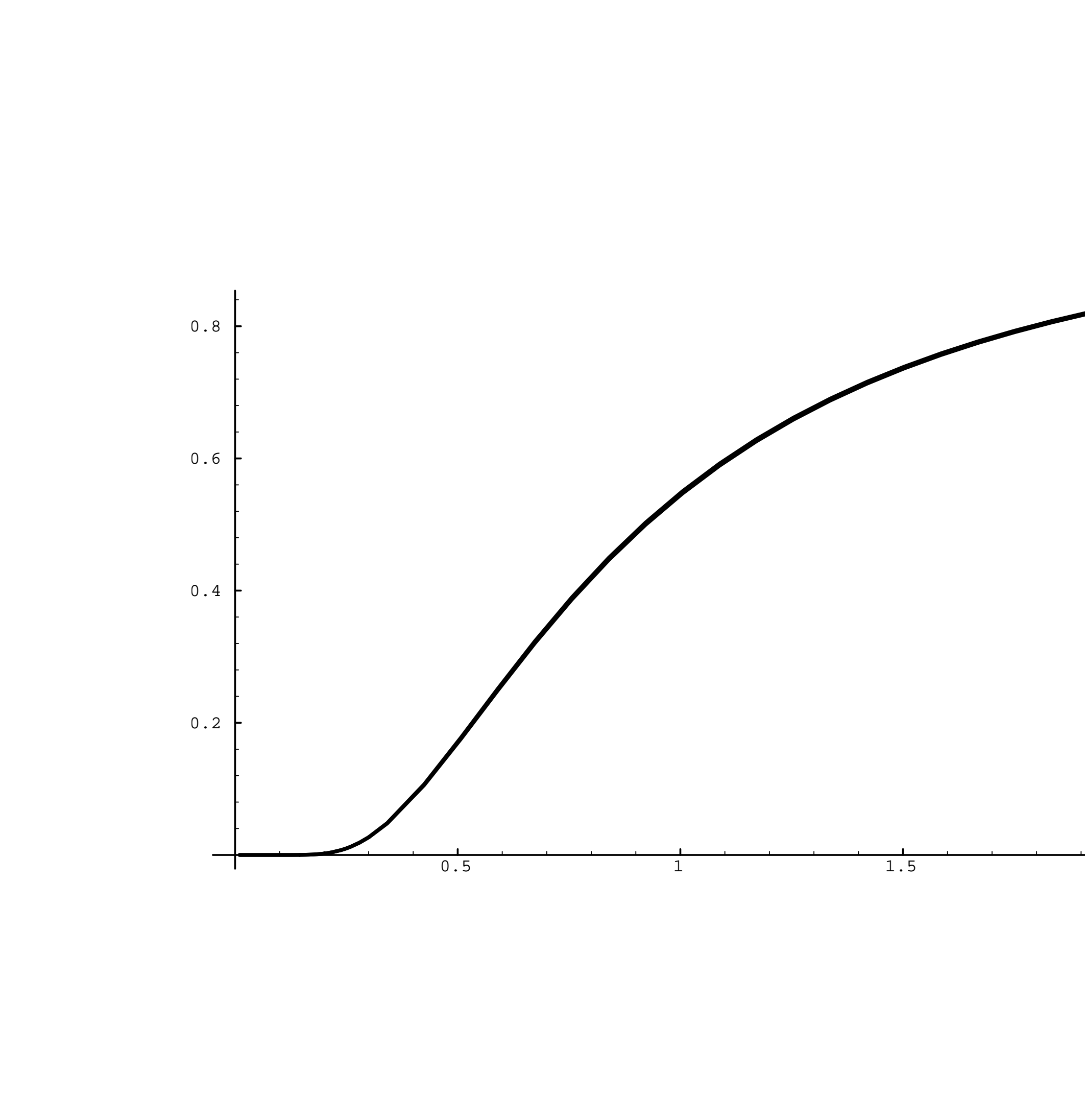




$$
1
$$

